\title{
The impact of chemotherapy-related nausea on patients' nutritional status, psychological distress and quality of life
}

\author{
Carole Farrell ${ }^{1}$, Sarah G Brearley ${ }^{2}$, Mark Pilling ${ }^{3}$, Alex Molassiotis ${ }^{3 *}$, \\ ${ }^{1}$ Christie NHS Foundation Trust, Manchester, UK \\ ${ }^{2}$ Faculty of Health and Medicine, Lancaster University, Lancaster, UK \\ ${ }^{3}$ School of Nursing, Midwifery \& Social Work, University of Manchester, Manchester, \\ UK
}

${ }^{*}$ Corresponding author contact details:

\author{
Alexander Molassiotis, RN, $\mathrm{PhD}$ \\ Professor of Cancer \& Supportive Care \\ University of Manchester \\ School of Nursing \\ University Place \\ MANCHESTER M13 9PL, \\ UK \\ email: alex.molassiotis@manchester.ac.uk \\ tel: +44 (0)161-3067830; fax: +44 (0)161-3067894
}

\section{Acknowledgements:}

This study was funded by the European Oncology Nursing Society through its Major Grant Award.

Conflict of Interest

Authors declare no conflict of interest. 
Abstract:

Purpose: Nausea is a troublesome and distressing symptom for patients receiving chemotherapy. While vomiting is well-controlled with current antiemetics, nausea is a more difficult symptom to manage. The aim of this study was to assess the impact of nausea on nutritional status, quality of life and psychological distress.

Methods: This was a prospective observational study over two cycles of chemotherapy. Patients completed the MASCC Antiemesis Tool, a measure of nutritional status (PG-SGA), the FACT-G quality of Life scale and the Hospital Anxiety \& Depression Scale at the end of each chemotherapy cycle (around day 10 post-chemotherapy).

Results: The sample consisted of 104 patients, primarily female, receiving anthracycline-based chemotherapy. While vomiting was minimal $(5.2-14.6 \%$ of patients), high levels of nausea were observed (55.2\%-72.9\%), and severe nausea (>6 on a $0-10$ scale) was reported by $20.5 \%-29.2 \%$ of the participants. Severe nausea had a borderline significant impact in relation to physical functioning $(p=0.025)$ and a significant impact on nutritional status (severe acute nausea, $p=0.003$; severe delayed nausea, $p=0.017$ ). Clinically meaningful changes were observed in relation to the FACT-G total score.

Conclusion: Chemotherapy induced nausea does have an impact on nutritional status and physical functioning and can impair anxiety and quality of life. As a key symptom associated with other symptoms it is imperative that greater attention is given to managing treatment-related nausea through innovative nonpharmacological and nutritional interventions.

Key words: nausea, chemotherapy, nutrition, anxiety, depression, quality of life, physical functioning 


\section{The impact of chemotherapy-related nausea on patients' nutritional status, psychological distress and quality of life}

\section{Background}

Chemotherapy-induced nausea is a significant problem in clinical practice, with 42$52 \%$ of patients experiencing nausea post-chemotherapy in routine practice $[15,25]$. Despite the availability of effective anti-emetics, chemotherapy-induced nausea and vomiting (CINV) is one of the most feared adverse events associated with chemotherapy $[8,12,37]$. The subjective and unobservable nature of CINV creates challenges in assessment [17,19] and means that clinicians tend to underestimate patients' experiences [17]. Generally, the clinical assessment of nausea is quite poor with clinical attention focused primarily on managing chemotherapy-induced vomiting rather than on the potential impact of chemotherapy-induced nausea. Unlike vomiting, nausea is more subjective and difficult for clinicians to evaluate and treat.

CINV can have a profoundly negative impact on social, physical and emotional functioning and on quality of life $[9,15,22]$. Moreover, despite recognition that nausea and vomiting are two related but separate entities, little attention has been directed to the concept of chemotherapy-induced nausea $[2,23]$. The impact of combined nausea and vomiting on quality of life $(\mathrm{QOL})$ has been highlighted in the literature. Osoba et al [30] in a study of 832 chemotherapy-naïve patients showed that those patients who had both nausea and vomiting had worse physical, cognitive and social functioning, global quality of life, fatigue, anorexia and dyspnoea compared to those who did not experience nausea and vomiting. Similarly, in a study of 119 patients receiving chemotherapy it was reported that those experiencing either nausea or vomiting had decreased $Q O L$ in several functioning and symptom subscales of the EORTC-QLQ-C30 scale [36]. A more recent study focusing on delayed nausea and vomiting also showed $\mathrm{QOL}$ impairments in patients experiencing these symptoms, highlighting that nausea had a stronger negative impact than vomiting on patients' daily lives [5]. Furthermore, there are indications that the presence of 'nutrition impact symptoms' that include nausea and impede oral intake are linked with poorer QOL [40]. 
Initial evidence from a study involving 220 lung cancer patients suggests that nausea forms a cluster of symptoms together with appetite loss, fatigue, weight loss, taste changes and vomiting [16]. Another study also highlighted that nausea, vomiting, feeling bloated, appetite loss, difficulty swallowing and taste changes are part of a gastrointestinal symptom cluster [27]. No work has focused to date on the nausea experience and nutritional impairment during chemotherapy, and establishing a link between nutritional symptoms and nausea seems imperative.

Hence, the aim of this study is to assess the impact of nausea on patients' nutritional status, quality of life and psychological distress.

\section{Methods}

A prospective observational study of patients over two cycles of chemotherapy treatment was undertaken using a quantitative descriptive exploratory design.

\section{Population and setting}

The study was carried out in a large cancer centre in the UK after approval from the local NHS Ethics and Research Committee. Consecutive patients were recruited if they were about to receive moderate or highly emetogenic adjuvant chemotherapy every three weeks as an outpatient and were willing to participate in the study. Patients were excluded if chemotherapy was palliative/disease stage was IV, if they had head and neck or upper gastrointestinal cancer, or if they were experiencing nausea from other causes.

Patients were recruited by a research assistant who provided detailed information about the study, and if patients agreed to participate they signed a consent form. Participants completed the study instruments prior to the first cycle of chemotherapy, at the end of cycle 1 and the end of cycle 2 and returned to the investigators either directly in clinic or using a pre-paid envelope.

\section{Study assessments}

Information about socio-demographic characteristics, type of cancer and chemotherapy regimens were obtained from the patients' medical records or the patients themselves. 
Nausea and vomiting was assessed using the Multinational Association of Supportive Care in Cancer (MASCC) Antiemesis Tool (MAT) [26]. This 8-item scale assesses presence (yes/no) and severity (0-10) of acute and delayed nausea, and presence (yes/no) and severity (number of times) of vomiting during chemotherapy Severe nausea was calculated based on the MAT visual analogue scale (VAS) score of 6-10 in the respective items. The MAT also defines nausea for the patients as 'the feeling that you might vomit'.

Nutritional status was assessed using the Patient Generated-Subjective Global Assessment (PG-SGA) [12,31]. This includes weight changes, alterations in food intake, gastrointestinal symptoms, changes in functional capacity and physical signs of malnutrition. Higher scores on the PG-SGA suggest a greater risk of malnutrition and scores of $\geq 9$ are indicative of malnutrition, in critical need of symptom improvement and/or nutritional intervention. In addition, objective measurements of weight, height, body mass index (BMI) and albumin levels were also recorded.

The Hospital Anxiety and Depression Scale (HADS) [42], a 14-item scale, was used to assess the presence and severity of anxiety and depression ( 7 items respectively). Scores $<8$ are within the normal range, scores of 8-10 indicate borderline cases needing further assessment before establishing psychopathology, and scores $11+$ indicate cases of clinical anxiety or depression respectively.

The Functional Assessment of Cancer Therapy-General (FACT-G) scale [7] was used to measure quality of life. This covers four dimensions of QOL: physical, social and family, emotional and functional well-being. The FACT-G questionnaire is scored using a 5 -point scale from $0=$ 'not at all' to $4=$ 'very much'.

\section{Data analysis}

Data was coded and entered into SPSS (v.15) and descriptive statistics used to summarize the data with missing values omitted from the calculation of 


\section{Results}

106 cancer patients participated in the study, however the analysis was undertaken on data from 104 patients, as 2 sets of questionnaires were incomplete and unusable. $92 \%$ completed all questionnaires at end of cycle 1 ( $n=96,8 \%$ attrition) and $85 \%$ at end of cycle 2 ( $n=88,15 \%$ attrition). Attrition was related primarily to the changing health status of the patients and being overwhelmed with the chemotherapy. Based on a power calculation for a bivariate test, in order to achieve an alpha of 0.05 and power of 0.80 , when the lowest correlation is 0.24 , an estimated number of 126 patients, as seen in past correlational studies, was required. In practise, only 104 patients were available for analysis which led to a small reduction in power $(=0.72)$.

The participants were mainly women (90.3\%, 93/103). 73\% (73/100) were married or with a partner, $58 \%$ were working and $25 \%$ had retired. The majority had completed secondary $(41.1 \%, 37 / 90)$ or college $(40 \%)$ education, while the remaining $18.9 \%$ had university or higher education. The mean age was 53.2 (SD 11.6, range=29-79). $83(80.6 \%, 83 / 103)$ patients had breast cancer, $10(9.7 \%)$ had bladder cancer and $8(7.8 \%)$ ovarian cancer. Chemotherapy regimens were mainly combinations of cytotoxic drugs: 81 (78.7\%) contained anthracyclines, $3(2.9 \%)$ contained taxanes, and 19 (18.5\%) were platinum-based. All patients received as primary antiemetics IV $8 \mathrm{mg}$ ondansetron with IV $8 \mathrm{mg}$ dexamethasone prior to chemotherapy administration and twice daily oral ondansetron (8mg) and 
dexamethasone $(4 \mathrm{mg})$ with oral metoclopramide $10 \mathrm{mg}$ (as required) for 2-3 days after chemotherapy.

\begin{abstract}
Nausea and Vomiting
MAT scores highlighted a decrease in acute vomiting between cycle $1(14.6 \%$, $14 / 96)$ and cycle $2(9.1 \%, 8 / 88)$ of chemotherapy, although the incidence of delayed vomiting increased slightly from 5.2 to $8.0 \%$ (5/96 to $7 / 87)$. However, the incidence of nausea was much greater with $55.2-64.8 \%$ of participants reporting acute nausea and $72.9-67.8 \%$ reporting delayed nausea after their first and second cycles of chemotherapy respectively [Figure 1].
\end{abstract}

\title{
INSERT FIGURE 1 HERE
}

The severity of acute and delayed vomiting was measured by the number of times participants vomited in the first 24 hours after chemotherapy. In terms of acute vomiting this ranged from one episode $(n=6 / 96,6.2 \%$ in cycle $1 ; n=6 / 88,6.8 \%$ in cycle 2 ), two episodes (5\% in cycle $1,2.3 \%$ in cycle 2 ), and three episodes $(3 \%$ in cycle $1,0 \%$ in cycle 2 ). In terms of delayed vomiting after cycle 1 this ranged from 0 8 episodes (mean $0.24, S D=1.17$ ). The duration was less after cycle 2 (range $0-5$ episodes, mean $0.17, \mathrm{SD}=0.70)$. In contrast, almost a third $(20.5-29.2 \%)$ of patients experienced severe nausea (either acute or delayed) [Figure 2].

\section{INSERT FIGURE 2 HERE}

\section{Nutritional status}

Baseline weight ranged from $50-125 \mathrm{~kg}$ (mean 74.05, SD 14.96) with little change over time and the BMl ranged from $19-42 \mathrm{~kg} / \mathrm{m}^{2}$ (mean 27.63, SD 5.06). Clinically, $27 / 85(32 \%)$ were of normal weight, 37 (44\%) overweight and 21 (25\%) were obese. Serum albumin was used to assess for possible malnutrition $(<35 \mathrm{~g} / \mathrm{dL})$ based on a normal reference range of $35-50 \mathrm{~g} / \mathrm{L}$. All participants but one had a serum albumin within the normal range at each time point.

Participants' scores on the PG-SGA indicated deteriorating nutritional status; $25.3 \%$ had symptoms of malnutrition after one cycle of chemotherapy and this was 
maintained across a further chemotherapy cycle [Table 1]. There was a statistically significant deterioration in PG-SGA scores from baseline (median=1) to the end of cycles 1 and 2 (median=5 and 4 respectively, $p<0.0001$ Friedman $\chi^{2}$ ). There is no trend in relation to presence of nausea and serum albumin levels. However, weight and $\mathrm{BMI}$ at cycle 1 has a trend to be lower for patients with poor nutritional status as can be seen in Table 1, although this did not reach statistical significance (ANOVA for weight and BMI respectively at cycle 2; $p=0.2, p=0.1$ ). On their own, however, albumin was lower after baseline (Friedman test, $p<0.001$ ), whereas weight was lower at the start of cycle $2(p=0.013)$ as it was for BMI $(p=0.006)$.

\section{INSERT TABLE 1 HERE}

\section{Psychological distress}

HADS scores showed variability in the range and mean scores at baseline, end of cycle 1 and end of cycle 2 chemotherapy . Levels of depression were quite low at baseline (score for clinical case of $>10=1.9 \% ; n=2 / 103$ ), although it showed a significant deterioration after baseline $(7.3 \%$ after cycle 1 [n=7/96] and $14.1 \%$ after cycle 2 [ $n=12 / 85])\left(p=0.0005\right.$, Friedman $\chi^{2}$ over time). In contrast, the incidence of anxiety was much higher at baseline $(n=19 / 102,18.6 \%)$, with a slight increase at the end of Cycle 1 ( $n=19 / 96,19.8 \%)$, although this change was not statistically significant.

\section{Quality of Life}

FACT-G total scores can range from 0-108, with higher scores indicating better QOL. Participants' total scores indicated deterioration in QOL after the start of chemotherapy. Mean scores were $83.68(\mathrm{SD}=14.5)$ at baseline, $77.8(\mathrm{SD}=17.7)$ at the end of cycle 1 and $78.4(\mathrm{SD}=16.4)$ at end of cycle 2; this change was statistically significant $\left(p=0.002\right.$, Friedman $\left.\chi^{2}\right)$.

\section{Impact of nausea on quality of life and nutritional status}

The proportion of patients at the end of cycle 1 who had a score $>=9$ in the PG-SGA, indicative of malnutrition, was higher in those experiencing acute nausea and delayed nausea $(58.3 \%$ and $79.2 \%$ respectively) than those who did not experience these symptoms $(41.7 \%$ and $20.8 \%$ respectively). This indicates a difference of $16.6 \%$ and $58.4 \%$ respectively in patients experiencing the two symptoms also having a PG-SGA 
score $>=9$. Similar data is also evident in relation to cycle 2 ( $72.7 \%$ vs. $27.3 \%$ for both distress at cycle $1(62.5 \%$ and $75 \%$ for acute and delayed nausea respectively vs. 37.5 and $25 \%$ ). Fisher's tests of difference for the above were all non-significant.

ANCOVA was used to identify significant associations between MAT nausea scores and QOL, nutritional status and psychological distress after cycle 1 with the level of significance set at 0.0125 due to testing four different binary groupings of the same patients. In general, baseline covariates were significant in the ANCOVA. Severe acute nausea had a borderline significant impact on physical QOL $(p=0.025)$, and a significant impact on nutritional status $(p=0.003$ for severe acute and $p=0.017$ for severe delayed nausea) [Table 3]. At cycle 2, ANCOVA calculations were not statistically significant for either acute or delayed nausea / severe nausea.

\section{INSERT TABLE 2 HERE}

\section{Discussion}

The findings of the present study clearly indicate that chemotherapy-induced nausea is associated with poor quality of life, nutritional status and psychological distress, with clinically meaningful differences being observed in those experiencing nausea compared to those who did not. This is the first study looking exclusively at the impact of chemotherapy-related nausea (rather than combined with vomiting as in past literature) on quality of life outcomes and one of the few focusing on nutritional aspects of nausea as a single entity. There was low incidence of acute vomiting after cycle $1(14.6 \%)$ and cycle $2(9.1 \%)$ of chemotherapy, with $5-8 \%$ of patients reporting delayed vomiting, reflecting that management of chemotherapy-induced vomiting has greatly improved over the last decade. In contrast, the incidence of chemotherapy-induced nausea was very high and severe nausea affected $20-30 \%$ of the patients over the two cycles of chemotherapy. However, these results should be viewed with some caution, as international antiemetic recommendations for anthracycline-based chemotherapy propose a three-drug combination, including aprepitant [35] and our sample received only two of them Plus metoclopramide); hence the incidence of nausea 
reported here may be over-estimated, although it reflects widely used routine clinical practices [25].

Although $20-30 \%$ of patients with cancer are known to experience psychological distress [21], this sample showed a low incidence of clinical depression (1.9-7.3\%) but higher scores of clinical anxiety (14.1-19.8\%). The psychological impact of chemotherapy is well recognised and associated with considerable uncertainty for patients [14]. However, clinical experience suggests patients' levels of anxiety decreases after the start of chemotherapy, which is reflected in these results. The borderline significant link with nausea highlights the distressing nature of this symptom.

Generally, while serum albumin levels were not significantly linked with nausea, patients' weight and BMI had a trend of decreasing from baseline to cycles 1 and 2 reflecting nutritional changes. Furthermore, the PG-SGA scores indicated deteriorating nutritional status after the start of chemotherapy, with $25 \%$ showing scores indicative of malnutrition after the first cycle of chemotherapy. This suggests that current standard measurements of nutritional status (weight and serum albumin) are inadequate indicators of malnutrition, and that clinicians should look to adopt alternative strategies to assess patients, such as the PGSGA. Body composition might change without reducing BMI and reflects the influence of nausea in nutritional status. Hence, BMI decreases and low serum albumin may not be sensitive indicators of malnutrition in this population which often experiences fluid retention during chemotherapy. Indeed, weight gain is common in women with breast cancer receiving chemotherapy [10,28,39]. Fat mass and lean body mass, components of body composition, are also difficult to measure in a clinical setting. The options are anthropometry using skin callipers measured at 4 sites and placed into an equation to calculate fat and fat free mass or bioelectrical impedance monitoring. The former is subject to a high degree of inter-rater reliability and requires some experience and training in the technique to produce valid results. The latter is subject to alterations in fluid balance, which would occur in chemotherapy patients. Therefore its usefulness would be of limited value and subject to strong opposition in relation to validity in oncology patients undergoing treatment. The Patient-generated SGA derived from the professional 
tool produced by Desksy [11] does incorporate a subjective assessment of body composition. This evaluates fat stores and muscle from pre-determined criteria that outline the visual assessment in some detail. The Patient-generated SGA is also validated against numerous nutritional status parameters, it has been used widely in oncology patients [3,32] and has been shown to be significantly associated with change in quality of life and change in lean body mass [4].

Generally, the nutritional impact of chemotherapy is poorly addressed by clinicians $[37,40]$; assessments are usually based on CTC toxicity scores that do not take into account important details of nutrition. If patients' weight generally remains stable and patients recover in between cycles of chemotherapy, current assessment approaches may fail to detect nutritional problems. This may be compounded by the lack of dieticians available for patients attending outpatient appointments. It is imperative that proactive nutritional assessment is integrated in clinical practice. Good nutrition practices may contribute to the relief of symptoms associated with nutrition (nausea, vomiting, diarrhoea, dysphagia, change in taste and smell, etc) and potentially improve quality of life, while poor nutrition may increase the incidence and severity of treatment side effects [1].

We have found that severe nausea has significant associations with nutritional status in this study, as judged by the proportions presented in table 3 and the statistically significant scores of the PG-SGA presented in table 4. As nausea is a key symptom whose presence (particularly severe acute and delayed nausea) has been demonstrated to be associated with decreased physical functioning and nutritional status, it is important to develop further research introducing nutritional interventions for the management of chemotherapy-related nausea. These may include education about what foods to eat/not to eat while nauseous, frequency, quantity, etc. This approach may be particularly appropriate, as the management of nausea is not satisfactory with current antiemetics.

Findings from this study showed that patients' QOL was sensitive to change within a short period of time, with some deterioration evident soon after the start of chemotherapy. Areas of impairments in quality of life such as physical functioning in those experiencing severe acute and delayed nausea confirm findings from past studies $[30,36]$, suggesting that little has changed in this area over the past $10-15$ 
years. While we have followed a conservative approach to statistical significance to decrease error in the results due to using multiple tests, there are indications that nausea, particularly severe nausea, has an association with functional, emotional and overall quality of life. This would need to be further ascertained in future research with the use of a larger and more heterogeneous sample. However, studies have established minimally important clinical change in relation to the FACT-G scale and in a sample with breast cancer patients this was around 5-6 points $[13,18]$. According to this estimate of clinically meaningful and important change, our results of the impact of nausea on patients' quality of life are clinically important. However, such estimates are not available for the nutritional and psychological distress outcome measures we have used.

Considering these data of unacceptably high levels of nausea and its clear association with quality of life and nutritional status, more interventions should be directed to the management of nausea. Evidence exists about the effects of acupuncture/acupressure, relaxation techniques, hypnosis, guided imagery, exercise, cognitive distraction, systematic desensitisation or behavioural approaches $[20,24,33,34]$ in managing nausea. Careful clinical management is also required to improve patients' concordance with treatment and prevent anticipatory nausea/vomiting developing in subsequent chemotherapy cycles.

Appropriate symptom management begins with symptom assessment and an accurate understanding of the prevalence and severity of symptoms experienced by patients. A number of instruments are available for the clinician to use, and critical reviews have provided evidence of their validity and usefulness, which clinicians could consult in order to select the most appropriate assessment tool for their practice [6, 41]. Furthermore, nausea can also be assessed as part of routine clinical practice when patients complete instruments that rapidly assess multiple symptoms.

This sample was mainly patients with breast cancer receiving anthracycline-based chemotherapy; therefore generalisability would be increased by using a more heterogeneous sample. As the sample is gender and breast cancer skewed, results may not be generalisable to males and other cancers. A larger sample may provide 
stronger evidence of the links between nausea and domains of quality of life, although our results are consistent with past literature using larger sample sizes but examining nausea and vomiting combined. The results are somewhat tentative, as the design of the study does not allow for a cause-and-effect outcome to be established, and the consequences of chemotherapy itself, stressors and other symptoms and comorbidities may also impact on the outcomes assessed in this study. Future research could focus on testing non-pharmacological interventions as adjunct therapies to antiemetics, improve the utility of available antiemetics by following evidence-based clinical guidelines and understand more fully the symptom of nausea from a pathophysiological perspective [29].

\section{Conclusions}

Our study confirms that nausea still remains a key quality of life problem for patients, it is distressing and leads to significant nutritional changes. This is clearly an important clinical problem, which this study assessed on its own rather than combined with the symptom of vomiting, recognising that nausea is related to vomiting but a different entity. The impact of nausea on nutritional status and quality of life is evident within days of chemotherapy administration. Given that most patients are treated in the outpatient setting, this requires careful assessment and clinical management. Specific nutritional interventions and use of a combination of pharmacological and non-pharmacological therapies for the management of this distressing symptom are necessary. 


\section{References}

1. American Cancer Society: Nutrition for the Person with Cancer: A Guide for Patients and Families. Atlanta, Ga: American Cancer Society, Inc., 2000.

2. ASHP (1999) Therapeutic guidelines on the pharmacologic management of nausea and vomiting in adult and pediatric patients receiving chemotherapy or radiation therapy or undergoing surgery. Am J Health Syst Pharm 56:729-764.

3. Bauer J CS, and Ferguson M: . Use of the scored Patient-Generated Subjective Global Assessment (PG-SGA) as a nutrition assessment Eur J Clin Nutr 2002;56:779-85.

4. Bauer JD CS. Nutrition intervention improves outcomes in patients with cancer cachexia receiving chemotherapy—a pilot study. Support Care Cancer 2005; 13:270-74.

5. Bloechl-Daum B, Deuson RR, Mavros P, Hansen M, Herrstedt J. Delayed nausea and vomiting continue to reduce patients' quality of life after highly and moderately emetogenic chemotherapy despite antiemetic treatment. J Clin Oncol 2006; 24: 4472-4478.

6. Brearley SG, Clements CV, Molassiotis A. A review of patient self-report tools for chemotherapy-induced nausea and vomiting. Support Care Cancer. 2008; 16: 1213-1229.

7. Cella D, Tulsky D, Gray G, Sarafian B, Linn E, Bonomi A et al (1993) The Functional Assessment of Cancer Therapy Scale : development and validation of the general measure. J Clin Oncol $11: 570-579$.

8. de Boer-Dennert M, de Wit R, Schmitz PI, Djontono J, v Beurden V, Stoter G \& Verweij J (1997) Patient perceptions of the side-effects of chemotherapy: the influence of 5HT3 antagonists. Br J Cancer 76:1055-1061.

9. Decker GM, DeMeyer ES, \& Kisko DL (2006) Measuring the maintenance of daily life activities using the functional living index-emesis (FLIE) in patients receiving moderately emetogenic chemotherapy. J Support Oncol 4:35-41

10. Demark-Wahnefried W, Peterson BL, Winer EP, Marks L, Aziz N, Marcom PK, Blackwell K, Rimer BK. Changes in weight, body composition, and factors influencing energy balance among premenopausal breast cancer patients receiving adjuvant chemotherapy. J Clin Oncol. 2001;19(9):2381-9. 
11.Detsky AS, Baker JP, Mendelson RA (1984). Evaluating the accuracy of nutritional assessment techniques applied to hospitalized patients: Methodology and comparisons. JPEN J Parenter Enteral Nutr. ;8(2):153-9.

12. Detsky AS, McLaughlin JR, Johnstone N, Whittaker S, Mendelson RA, Jeejeebhoy KN (1987) What is subjective global assessment of nutritional status. JPEN J Parenter Enteral Nutr 11:8-13.

13. Eton DT, Cella D, Yost KJ, et al. (2004). A combination of distribution- and anchor-based approaches determined minimally important differences (MIDs) for four endpoints in a breast cancer scale. J Clin Epidemiol.; 57(9):898-910.

14. Farrell C, Heaven C, Beaver K, Maguire P (2005). Identifying the concerns of women undergoing chemotherapy. Patient Education and Counseling, 56: 72-7.

15. Glaus A, Knipping C, Morant R, Bohme C, Lebert B, Beldermann F, Glawogger B, Ortega PF, Husler A \& Deuson R (2004) Chemotherapy-induced nausea and vomiting in routine practice: a European perspective. Support Care Cancer 12:708-715.

17.16. Gift AG, Jablonski A, Stommel M, Given CW (2004) Symptom clusters on elderly patients with lung cancer. Oncol Nurs Forum 31 :203-212. Grunberg SM, Deuson RR, Mavros P, Geling O, Hansen M, Cruciani G, Daniele B, De Pouvourville G, Rubenstein EB \& Daugaard G (2004) Incidence of chemotherapy-induced nausea and emesis after modern antiemetics. Cancer 100:2261-2268.

18. King MT, Stockler MR, Cella DF, et al. (2010). Meta-analysis provides evidencebased effect sizes for a cancer-specific quality-of-life questionnaire, the FACTG. J Clin Epidemiol.; 63(3):270-281.

19. Liau CT, Chu NM, Liu HE, Deuson R, Chen JS (2005) Incidence of chemotherapy-induced nausea and vomiting in Taiwan: physicians' and nurses' estimation vs patient's reported outcomes. Supp Care Cancer 13:277-286.

20. Lotfi-Jam K, Carey M, Jefford M, Schofield P, Charleson C, Aranda S. Nonpharmacologic strategies for managing common chemotherapy adverse effects: a systematic review. J Clin Oncol. 2008; 26: 5618-5629

21. Maguire P (2000). Managing psychological morbidity in cancer patients. European Journal of Cancer 36: 556-58. 
22. Martin CG, Rubenstein EB, Elting LS, Kim YJ \& Osoba D (2003) Measuring chemotherapy-induced nausea and emesis. Psychometric properties of a quality of life questionnaire. Cancer 98:645-655.

23. Miller M, Kearney N (2004) chemotherapy-related nausea and vomiting- past reflections, present practice and future management. Eur J Cancer Care 13:7181.

24. Molassiotis A, Yung HP, Yam BM, Chan FY, Mok TS. The effectiveness of progressive muscle relaxation training in managing chemotherapy-induced nausea and vomiting in Chinese breast cancer patients: a randomised controlled trial. Support Care Cancer. 2002; 10: 237-246.

25. Molassiotis A, Saunders M, Valle J, Wilson G, Lorigan P, Wardley A, Levine E, Cowan R, Loncaster J \& Rittenberg C (2008) A Prospective observational study of chemotherapy-induced nausea and vomiting in routine practice in a UK cancer centre. Support Care Cancer 16: 201-208.

26. Molassiotis A, Coventry PA, Stricker CT, Clements C, Eaby B, Velders L, Rittenberg C \& Gralla RJ (2007) Validation and psychometric assessment of a short clinical scale to measure chemotherapy-induced nausea and vomiting: the MASCC Antiemesis Tool (MAT). J Pain Symptom Manage 34:148-159.

27. Molassiotis A, Wengström $Y$, Kearney N. Symptom cluster patterns during the first year after diagnosis with cancer. J Pain Symptom Manage. 2010; 39: 847858.

28. Nissen MJ, Shapiro A, Swenson KK. Changes in weight and body composition in women receiving chemotherapy for breast cancer. Clin Breast Cancer. 2011; $11(1): 52-60$.

29. Olver I, Molassiotis A, Aapro M, Herrstedt J, Grunberg S, Morrow G. Antiemetic research: future directions. Support Care Cancer. 2011; 19 Suppl 1: S49-55.

30. Osoba D, Zee B, Warr D, Latreille J, Kaizer L, Pater J. Effect of postchemotherapy nausea and vomiting on health-related quality of life. The Quality of Life and Symptom Control Committees of the National Cancer Institute of Canada Clinical Trials Group.Suport Care Cancer 1997; 5: 307-313.

31. Ottery F (2000) Patient-generated subjective global assessment. In Polisena C (ed) The clinical guide to oncology nutrition, American Dietetic Association: Chicago, pp. 1-23. 
32. Read JA, Crockett N, Volker DH, MacLennan P, Choy STB, Beale P, et al. Nutritional Assessment in Cancer: Comparing the Mini-Nutritional Assessment (MNA) With the Scored Patient-Generated Subjective Global Assessment (PGSGA). Nutrition and Cancer 2005;53(1):51-56.

33. Richardson J, Smith JE, McCall G, Richardson A, Pilkington K, Kirsch I. Hypnosis for nausea and vomiting in cancer chemotherapy: a systematic review of the research evidence. Eur J Cancer Care 2007; 16: 402-412.

34. Roscoe JA, Morrow GR, Hickok JT, Bushunow P, Pierce HI, Flynn PJ, Kirshner JJ, Moore DF, Atkins JN. The efficacy of acupressure and acustimulation wrist bands for the relief of chemotherapy-induced nausea and vomiting. A University of Rochester Cancer Center Community Clinical Oncology Program multicenter study. J Pain Symptom Manage. 2003; 26: 731-742.

35. Roila F, Herrstedt J, Aapro M, Gralla RJ, Einhorn LH, Ballatori E, Bria E, ClarkSnow RA, Espersen BT, Feyer P, Grunberg SM, Hesketh PJ, Jordan K, Kris MG, Maranzano E, Molassiotis A, Morrow G, Olver I, Rapoport BL, Rittenberg C, Saito M, Tonato M, Warr D; ESMO/MASCC Guidelines Working Group. Guideline update for MASCC and ESMO in the prevention of chemotherapyand radiotherapy-induced nausea and vomiting: results of the Perugia consensus conference. Ann Oncol. 2010; 21 Suppl 5:v232-43.

36. Rusthoven JJ, Osoba D, Butts CA, Yelle L, Findlay H, Grenville A. The impact of postchemotherapy nausea and vomiting on quality of life after moderately emetogenic chemotherapy. Support Care Cancer 1998; 6: 389-395.

37. Shaw C. Nutrition and cancer. Blackwell Publishers, Oxford, UK, 2011.

38. Smith HS (2005) A receptor-based paradigm of nausea and vomiting. J Cancer Pain Symptom Pall 1:11-23.

39. Trédan O, Bajard A, Meunier A, Roux P, Fiorletta I, Gargi T, Bachelot T, Guastalla JP, Lallemand Y, Faure C, Pérol D, Bachmann P. Body weight change in women receiving adjuvant chemotherapy for breast cancer: a French prospective study. Clin Nutr. 2010;29(2):187-91.

40. Tong $H$, Isenring $E$, Yates $P$. The prevalence of nutrition impact symptoms and their relationship to quality of life and clinical outcomes in medical oncology patients. Support Care Cancer 2009; 17: 83-90.

41. Wood JM, Chapman K, Eilers J. Tools for assessing nausea, vomiting, and retching. Cancer Nurs. 2011; 34: E14-24. 
42.Zigmund A S \& Snaith R P. (1983) Hospital Anxiety and Depression Scale.

1 Acta Psych Scand 67: 361-370. 
Figure 1: MAT Acute and delayed nausea and vomiting ( $\%$ of 'Yes' answers) with Exact upper $95 \% \mathrm{Cl}$ bars. Cycle1 $=96$ responses. Cycle2=88 acute, 87 delayed responses.

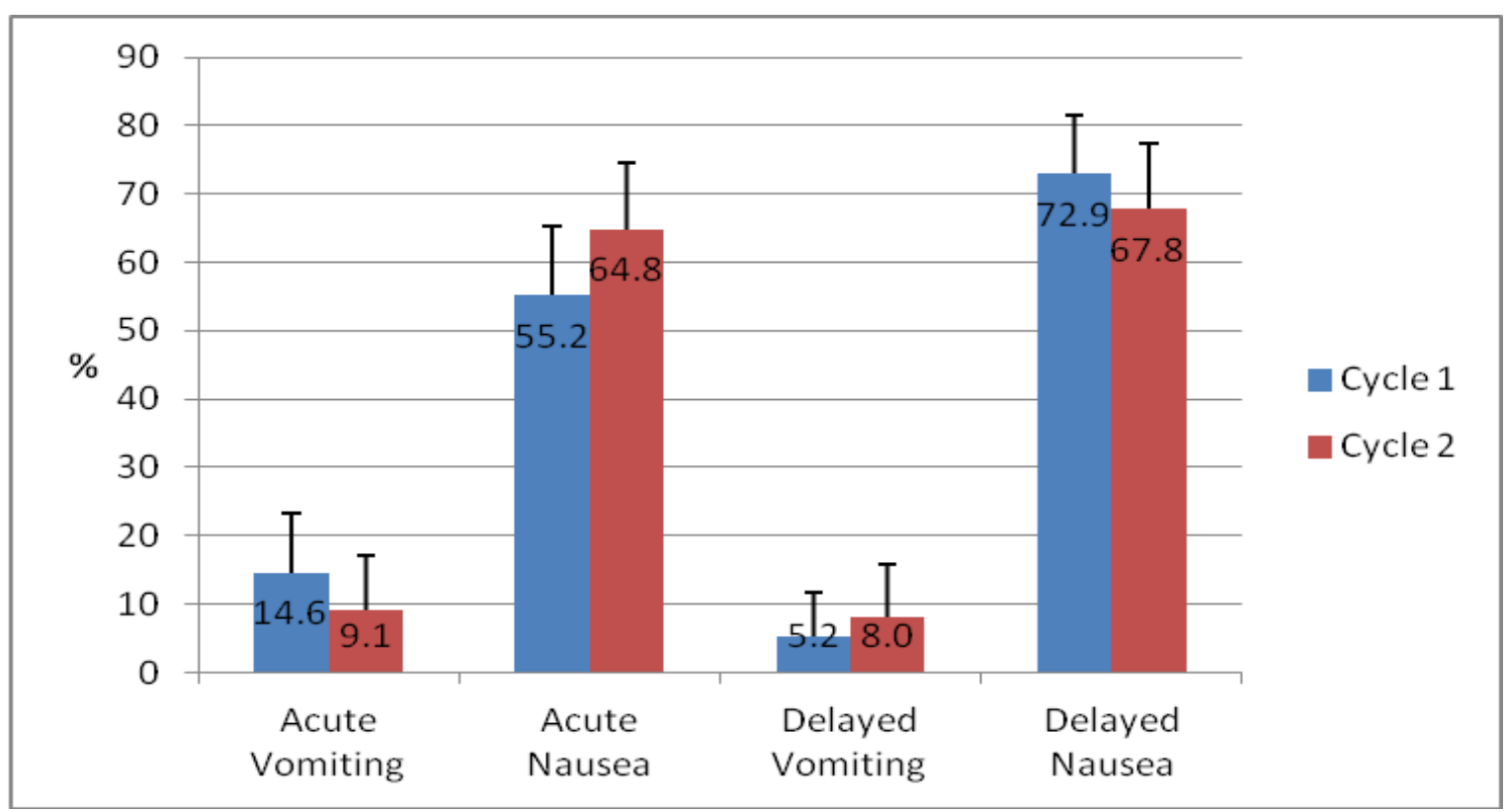


Figure 2: Incidence of severe nausea (6-10 on the MAT item of nausea) (\% of patients), with Exact upper $95 \% \mathrm{Cl}$ bars. Cycle1 $=96$ responses. Cycle2=88 acute, 87 delayed responses.

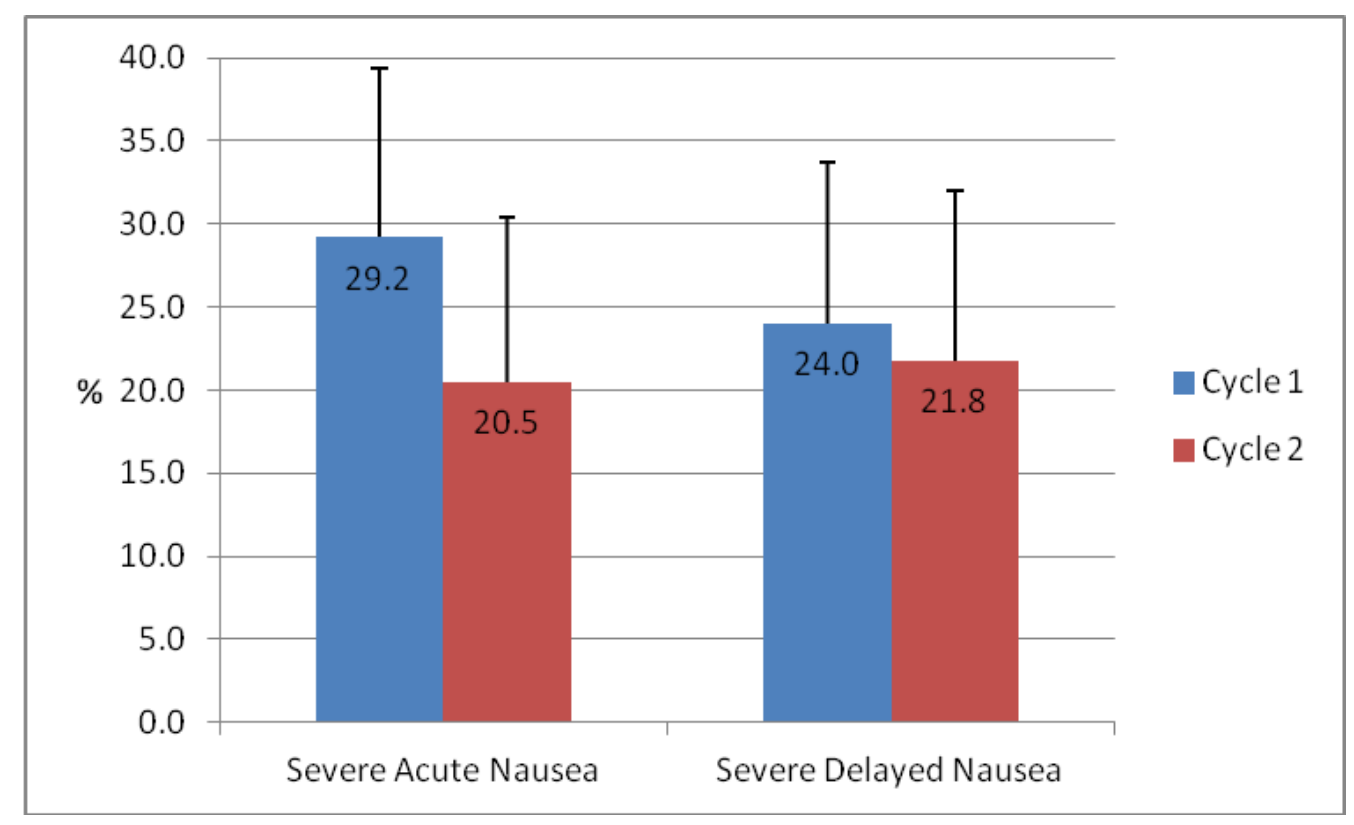


Table 1: Nutritional status indicators: changes over time

\begin{tabular}{|l|ll|ll|}
\hline & Median & Total & $\begin{array}{l}\text { PG-SGA } \\
\text { Score 0- 8 } \\
\text { n (\%) }\end{array}$ & $\begin{array}{l}\text { PG-SGA } \\
\text { Score } \mathbf{9} \\
\text { n (\%) }\end{array}$ \\
\hline PG-SGA Baseline & 1 & 101 & $91(90.1)$ & $10(9.9)$ \\
PG-SGA Cycle 1 & 5 & 95 & $71(74.7)$ & $24(25.3)$ \\
PG-SGA Cycle 2 & 4 & 87 & $65(74.7)$ & $22(25.3)$ \\
\hline & Median & Total & Median & Median \\
\hline Weight at Baseline & 72.0 & 96 & 72 & 72.1 \\
Weight pre Cycle1 & 71.7 & 87 & 71.7 & 72.7 \\
Weight pre Cycle2 & 72.7 & 42 & 72.8 & 64.5 \\
\hline BMI at Baseline & 27.4 & 85 & 27.5 & 28.5 \\
BMI pre Cycle1 & 27.1 & 74 & 26.7 & 27.5 \\
BMI pre Cycle2 & 26.1 & 37 & 26.4 & 24.4 \\
\hline Albumin at Baseline & 43 & 95 & 43 & 45 \\
Albumin pre Cycle1 & 42 & 93 & 42.5 & 42 \\
Albumin pre Cycle2 & 42 & 87 & 42 & 43 \\
\hline
\end{tabular}


Table 2: $\quad$ Mean scores of Quality of Life, nutritional status and psychological distress in relation to acute and delayed nausea (ANCOVA analysis) for Cycle $1(n=96)$. Patients are analysed in four different binary groupings.

\begin{tabular}{|c|c|c|c|c|c|c|c|c|}
\hline & \multicolumn{2}{|c|}{ Acute Nausea } & \multicolumn{2}{|c|}{$\begin{array}{ll}\text { Severe } & \text { Acute } \\
\text { Nausea } & \end{array}$} & \multicolumn{2}{|c|}{ Delayed Nausea } & \multicolumn{2}{|c|}{$\begin{array}{l}\text { Severe } \\
\text { Nausea }\end{array}$} \\
\hline & No & yes & $<6$ & $>=6$ & No & yes & $<6$ & $>=6$ \\
\hline $\begin{array}{l}\text { FACT-G: } \\
\text { Physical }\end{array}$ & 20.5 & 17.8 & 20.2 & 16.1 & 20.7 & 18.3 & 20.1 & 15.5 \\
\hline $\begin{array}{l}\text { FACT-G: } \\
\text { Social }\end{array}$ & 24.0 & 24.3 & 24.2 & 24.0 & 23.6 & 24.4 & 24.1 & 24.3 \\
\hline $\begin{array}{l}\text { FACT-G: } \\
\text { Emotional }\end{array}$ & 19.0 & 17.0 & 18.4 & 16.6 & 18.8 & 17.6 & 18.1 & 17.4 \\
\hline $\begin{array}{l}\text { FACT-G: } \\
\text { Functional }\end{array}$ & 17.4 & 15.8 & 17.2 & 15.0 & 17.1 & 16.3 & 17.2 & 14.2 \\
\hline $\begin{array}{l}\text { FACT-G: } \\
\text { Total }\end{array}$ & 80.9 & 75.0 & 80.1 & 71.8 & 80.2 & 76.8 & 79.5 & 71.8 \\
\hline$P G-S G A$ & 5.0 & 6.5 & 5.0 & 8.0 & 4.3 & 6.4 & 5.1 & 8.0 \\
\hline $\begin{array}{l}\text { HADS: } \\
\text { Anxiety }\end{array}$ & 4.8 & 7.2 & 5.7 & 7.1 & 5.5 & 6.3 & 5.8 & 7.0 \\
\hline $\begin{array}{l}\text { HADS: } \\
\text { Depression }\end{array}$ & 4.0 & 5.2 & 4.5 & 5.2 & 4.7 & 4.6 & 4.6 & 5.0 \\
\hline $\begin{array}{l}\text { HADS: } \\
\text { Total }\end{array}$ & 8.8 & 12.3 & 10.1 & 12.3 & 10.2 & 11.0 & 10.4 & 12.0 \\
\hline
\end{tabular}




\begin{tabular}{|l|l|l|l|l|l|l|l|l|}
\hline & $\begin{array}{l}\text { Difference } \\
\text { in mean }\end{array}$ & $\mathbf{P}$ value & $\begin{array}{l}\text { Difference } \\
\text { in mean }\end{array}$ & $\mathbf{P}$ value & $\begin{array}{l}\text { Difference } \\
\text { in mean }\end{array}$ & P value & $\begin{array}{l}\text { Difference } \\
\text { in mean }\end{array}$ & P value \\
\hline $\begin{array}{l}\text { FACT-G: } \\
\text { Physical }\end{array}$ & 2.7 & 0.052 & 4.1 & $0.025^{*}$ & 2.4 & 0.397 & 4.6 & 0.217 \\
\hline $\begin{array}{l}\text { FACT-G: } \\
\text { Social }\end{array}$ & -0.3 & 0.518 & 0.2 & 0.248 & -0.8 & 0.185 & -0.2 & 0.038 \\
\hline $\begin{array}{l}\text { FACT-G: } \\
\text { Emotional }\end{array}$ & 2 & 0.907 & 1.8 & 0.193 & 1.2 & 0.754 & 0.7 & 0.595 \\
\hline $\begin{array}{l}\text { FACT-G: } \\
\text { Functional }\end{array}$ & 1.6 & 0.675 & 2.2 & 0.412 & 0.8 & 0.682 & 3 & 0.409 \\
\hline $\begin{array}{l}\text { FACT-G: } \\
\text { Total }\end{array}$ & $5.9 \dagger$ & 0.603 & $8.3 \dagger$ & 0.566 & 3.4 & 0.907 & $7.7 \dagger$ & 0.628 \\
\hline PG-SGA & -1.5 & 0.05 & -3 & $0.003^{* *}$ & -2.1 & 0.051 & -2.9 & $0.017^{*}$ \\
\hline $\begin{array}{l}\text { HADS: } \\
\text { Anxiety }\end{array}$ & -2.4 & 0.494 & -1.4 & 0.978 & -0.8 & 0.961 & -1.2 & 0.638 \\
\hline $\begin{array}{l}\text { HADS: } \\
\text { Depression }\end{array}$ & -1.2 & 0.579 & -0.7 & 0.794 & 0.1 & 0.405 & -0.4 & 0.554 \\
\hline $\begin{array}{l}\text { HADS: } \\
\text { Total }\end{array}$ & -3.5 & 0.592 & -2.2 & 0.74 & -0.8 & 0.547 & -1.6 & 0.429 \\
\hline
\end{tabular}

** Statistically significant $(<5 \% / 4=0.0125)$

* Borderline statistically significant $(<5 \%)$

† FACT-G scale: Clinically meaningful change, although not statistically significant 